\title{
The Predicted Metabolic Function of the Gut Microbiota of Drosophila melanogaster
}

Nana Y.D. Ankrah ${ }^{1,6 \#}$, Brandon E. Barker ${ }^{2}$, Joan Song ${ }^{3,7}$, Cindy Wu ${ }^{4}$, John G. McMullen II $^{1}$ and Angela E. Douglas ${ }^{1,5}$

${ }^{1}$ Department of Entomology, ${ }^{2}$ Center for Advanced Computing, ${ }^{3}$ School of Electrical and Computer Engineering, ${ }^{4}$ Robert Frederick Smith School of Chemical and Biomolecular Engineering, and ${ }^{5}$ Department of Molecular Biology and Genetics, Cornell University, Ithaca, New York, USA

${ }^{6}$ Current address: Department of Biological Sciences, State University of New York at Plattsburgh, Plattsburgh, NY, USA

${ }^{7}$ Current Address: The Medical Scientist Training Program, Albert Einstein College of Medicine, New York, NY, USA.

Running title: Drosophila gut microbiome metabolic function \# Corresponding Author

Nana Y.D. Ankrah, Department of Biological Sciences, State University of New York at Plattsburgh, 101 Broad Street, Plattsburgh, NY 12901, USA. Email:nankr001@plattsburgh.edu 


\section{ABSTRACT}

2 An important goal for many nutrition-based microbiome studies is to identify the metabolic

3 function of microbes in complex microbial communities and its impact on host physiology. This

4 research can be confounded by poorly-understood effects of community composition and host

5 diet on the metabolic traits of individual taxa. Here, we investigated these multi-way interactions

6 by constructing and analyzing metabolic models comprising every combination of five bacterial

7 members of the Drosophila gut microbiome (from single taxa to the five-member community of

8 Acetobacter and Lactobacillus species) under three nutrient regimes. We show that the

9 metabolic function of Drosophila gut bacteria is dynamic, influenced by community composition

10 and responsive to dietary modulation. Furthermore, we show that ecological interactions such as

11 competition and mutualism identified from the growth patterns of gut bacteria are underlain by a

12 diversity of metabolic interactions, and show that the bacteria tend to compete for amino acids

13 and B vitamins more frequently than for carbon sources. Our results reveal that in addition to

14 fermentation products such as acetate, intermediates of the tricarboxylic acid (TCA) cycle

15 including 2-oxoglutarate and succinate are produced at high flux and cross-fed between bacterial

16 taxa suggesting important roles for TCA cycle intermediates in modulating Drosophila gut

17 microbe interactions and the potential to influence host traits. These metabolic models provide

18 specific predictions of the patterns of ecological and metabolic interactions among gut bacteria

19 under different nutrient regimes, with potentially important consequences for overall community

20 metabolic function and nutritional interactions with the host. 


\section{IMPORTANCE}

23 Drosophila is an important model for microbiome research partly because of the low complexity

24 of its mostly culturable gut microbiota. Our current understanding of how Drosophila interacts

25 with its gut microbes and how these interactions influence host traits derives almost entirely from

26 empirical studies that focus on individual microbial taxa or classes of metabolites. These studies

27 have failed to capture fully the complexity of metabolic interactions that occur between host and

28 microbe. To overcome this limitation, we reconstructed and analyzed 31 metabolic models for

29 every combination of the five principal bacterial taxa in the gut microbiome of Drosophila. This

30 revealed that metabolic interactions between between Drosophila gut bacterial taxa are highly

31 dynamic and influenced by co-occurring bacteria and nutrient availability. Our results generate

32 testable hypothesis about among-microbe ecological interactions in the Drosophila gut and the

33 diversity of metabolites available to influence host traits.

34

35 Key words: microbiome, constraint-based modeling, mutualism, competition, cross-feeding,

36 Drosophila 


\section{INTRODUCTION}

38 Microbiomes associated with animals are variable in taxonomic composition and impact on host

39 traits (1-6). This has generated two linked challenges: to understand the causes of the variability

40 and to predict how the microbiome may interact with other variables, such as host genotype, age,

41 and activity, as well as diet, to determine host traits (7-11). Strategies to reduce and manage

42 these highly complex interactions include the use of simplified microbial communities of defined

43 taxonomic composition (12-14), and modeling to investigate patterns across larger scales of

44 parameter space than is technically feasible by empirical study (15-19).

The gut microbiota of Drosophila is an attractive system to study taxonomically simple

47 microbiomes because its microbiome is naturally of low diversity, generally with fewer than 20

48 bacterial species in laboratory culture, and microbiomes of defined composition can readily be

49 generated and maintained $(20,21)$. In most laboratory cultures, the microbiome is dominated by

50 acetic acid bacteria (AABs), generally of the genus Acetobacter ( $\alpha$-proteobacteria), and lactic

51 acid bacteria (LABs) of the genus Lactobacillus (Firmicutes) (20), although some studies have

52 reported strong representation of other taxa, including $\gamma$-proteobacteria (e.g. Stenotrophomonas

53 spp.) and Lactobacilli (e.g. Enterococcus spp.) (22, 23). Studies on associations with a single

54 bacterial isolate and with communities of 2-5 microbial taxa have demonstrated that both

55 individual taxa and communities can, variously, contribute to B vitamin nutrition, reduce lipid

56 content, influence development rates, lifespan and fecundity, and modulate olfactory and egg-

57 laying behavior $(14,24-30)$. In several studies, these microbiome effects on host traits have been

58 shown to vary with diet composition, and they have been linked to among-microbe interactions

59 that influence both the abundance and metabolic activity of individual microbial taxa $(14,25,29-$ 
60 31). However, the relationship between diet, community composition and metabolic function of

61 the microbiome remains poorly-understood.

63 The goal of this study was twofold: first, to determine the combined effects of community

64 composition and nutrient availability on the metabolic interactions among members of the

65 Drosophila gut microbiota; and second to establish how these interactions shape the abundance

66 of the microorganisms in the community and the production of metabolites that may influence

67 host traits. We adopted a modeling approach, specifically to construct and analyze the metabolic

68 model for every combination of 5 bacterial taxa isolated from the Drosophila gut microbiome:

69 Acetobacter fabarum, A. pomorum, A. tropicalis, Lactobacillus brevis, and L. plantarum. This

70 choice of taxa enabled us to examine interactions among species of different taxonomic

71 relatedness and metabolic function: within the Acetobacteraceae, the closely-related $A$. fabarum

72 and A. pomorum are assigned to a different subgroup of Acetobacter from A. tropicalis (32); and

73 the homofermentative L. plantarum and heterofermentative L. brevis are members of different

74 phylogenetic groups of Lactobacillus (33). Members of the genus Acetobacter and Lactobacillus

75 are well represented across both field and lab Drosophila populations (34-37) and have been

76 widely used to investigate the impact of gut microbes on host physiology $(14,38-40)$. The

77 metabolic network for each species was reconstructed from annotated metabolism genes in the

78 sequenced genome, and the networks for the different species in each community integrated into

79 community models. We then applied the SteadyCom framework (41) to quantify the steady-state

80 composition of each community and to predict the metabolic flux within and between the

81 bacteria contributing to each community. We used SteadyCom because other community

82 modeling approaches generally assume fixed community composition lack constraints that 
83 prevent fast-growing organisms from displacing other microbes in the community regardless of

84 nutrient availability in their environment. SteadyCom applies more ecologically relevant

85 constraints and imposes a single, time-averaged constant growth rate across all members of a

86 community to ensure co-existence and stability as predicted to occur in animal guts. SteadyCom

87 is also applicable to established constraint-based modeling approaches such as flux variability

88 analysis, an important tool for determining the robustness of metabolic models in various

89 simulation conditions. This approach enabled us to assess how community composition is

90 influenced by antagonistic and mutualistic metabolic interactions, and to evaluate how among-

91 microbe interactions can dictate overall metabolic outputs from the community.

\section{RESULTS}

94 Growth of Drosophila gut bacterial communities under different nutrient regimes. Our first

95 analysis tested for growth in silico of the 31 possible communities of the five test bacteria under

96 three nutrient regimes (Fig. 1). As predicted, all five single-species communities displayed

97 growth in both the base medium, comprising the complete set of nutrients required for growth by

98 all the bacteria, and the nutrient-rich medium, in which the complete set of nutrients was

99 provided in excess. However, only two bacteria, the acetic acid bacteria (AAB) A. pomorum and

100 A. tropicalis, grew on the minimal medium containing glucose, glycerol, ammonia, sulfate, and

101 phosphate as primary sources of carbon, nitrogen, sulfur, and phosphorus respectively. Growth

102 of the third AAB, A. fabarum was rescued by co-culture with any other AAB or with one of the

103 two lactic acid bacteria (LAB), L. plantarum, but not with L. brevis. Similarly, growth of $L$.

104 plantarum was rescued by co-culture with any AAB but not with L. brevis. The co-culture

105 requirements of $L$. brevis were greater, requiring both L. plantarum and at least one AAB. The 
failure of A. fabarum, L. plantarum, and L. brevis to grow in silico on the minimal medium was a

107 consequence of their auxotrophy for amino acids such as arginine and the peptidoglycan

108 precursor diaminoheptanedioate. These metabolites are released from bacterial species, e.g. $A$.

109 pomorum and $A$. tropicalis, that rescue growth.

111 Further inspection of the data in Fig. 1 revealed the diverse effects of co-culture on growth

112 dynamics of individual bacteria. For example, co-culture with $A$. tropicalis tended to reduce the

113 growth of the other bacteria on the nutrient-rich medium; and the growth of AABs on the

114 minimal medium was largely unaffected by co-culture with L. plantarum in two-member

115 communities, but strongly repressed in communities of two or three AABs with L. plantarum

116 (Table S1). To investigate these effects systematically, we classified co-culture interactions as

117 sets of binary interactions: 1) competitive if both organisms displayed reduced growth in co-

118 culture, 2) parasitic if the growth of one member was enhanced at the expense of another, 3)

119 mutualistic if both organisms displayed increased growth in co-culture, 4) commensal if one

120 organism displayed increased growth with no change in the other, 5) amensal if one organism

121 displayed reduced growth with no change in the other, and 6) neutral if the growth of both

122 organisms was unaltered. Increases and decreases in growth were determined by comparing the

123 growth of a microbe in isolation to its growth in co-culture. In the nutrient-rich and basal media,

124 the interactions were exclusively antagonistic: $50-70 \%$ of the interactions were competitive, and

125 the remainder were parasitic (Fig. 2A, Table S1). In the minimal medium, parasitic interactions

126 predominated, but competitive, mutualistic, amensal and neutral interactions also occurred (Fig.

127 2A). Notably, mutualistic interactions accounted for $12 \%$ of the interactions in two-member

128 communities and increased progressively to $30 \%$ of the interactions in the five-member 
129 community. A large proportion ( $\sim 80 \%)$ of neutral interactions occur between A. fabarum and L.

130 brevis and these interactions switch to mutualistic interactions when L. plantarum is added to the

131 community (Table S1C). Similarly, $\sim 66 \%$ of amensal interactions occur between L. brevis and

132 an at least one AAB (Table S1C). L. brevis-AAB amensal interactions switch to parasitic (L.

133 brevis growth increases, AAB growth decreases) in more complex communities with the

134 addition of L. plantarum. The switch in interaction type is facilitated by L. plantarum production

135 of meso-2,6-diaminoheptanediote, an essential metabolite for L. brevis growth.

137 Patterns of metabolite consumption and release. We hypothesized that the competitive

138 interactions in the simulated bacterial communities (Fig. 2A) was underlain by the co-

139 consumption of individual nutrients by two or more bacteria in the community; these nutrients

140 may be constituents of the medium or, in communities with three or more members, derived

141 from other bacteria. We, additionally, hypothesized that parasitic interactions involved the

142 unidirectional cross-feeding of metabolites from the bacterium displaying depressed growth to

143 the bacterium displaying increased growth in co-culture; while mutualistic interactions involved

144 the reciprocal transfer of metabolites that were synthesized and released by one bacterium and 145 required for growth by the other bacterium.

147 For our first analysis of the incidence of competition, we quantified the number of individual 148 metabolites consumed by more than one bacterium in the simulated communities (Figure 2B,

149 Table S2A-C, Dataset S1). As shown in Fig. 2B, the number of nutrients shared between two

150 bacteria increased with both community complexity and nutrient content of the growth media.

151 The number of shared metabolites increased significantly between two-member and three- 
152 member communities but increases in three to four and four to five member communities were

153 mostly not significant for all media types (Table S2D). Consistent with the relatively low

154 incidence of competitive interactions in the minimal medium, the greatest number of overlapping

155 input metabolites recorded for any interaction in this medium was 12, which was half or less of

156 the equivalent values, 21 and 29 for the basal medium and nutrient-rich medium, respectively

157 (Fig. 2B).

159 To investigate the specific metabolic drivers of the antagonistic growth interactions, i.e. both

160 competition and parasitism, we determined the input and output metabolites of each bacterium in

161 every community. A total of 100 unique metabolites was predicted to be produced or consumed.

162 We classified each metabolite by the frequency of its consumption by members of the gut

163 community (Fig. 3, Table S3A-C). Our data show distinct metabolite use patterns associated

164 with competitive, parasitic and mutualistic growth outcomes. Competitive growth interactions

165 were significantly dominated by single-use or co-consumption in the rich and base media, but

166 not in the minimal medium (Fig. 3A-C, Table S3D). Similarly, parasitic interactions were

167 significantly dominated by single-use or co-consumption in rich and base media, and single-

168 produced, and cross-fed metabolite use patterns dominated minimal medium parasitic

169 interactions (Fig. 3D-F, Table S3D). Mutualistic growth interactions, observed only in the

170 minimal medium, were characterized by the dominance of cross-feeding interactions which made

171 up a significantly larger proportion of all interaction types (Fig. 3G, Table S3D).

172

173 Our data also show shifts in metabolite use profiles with depletion of nutrients in the growth

174 medium. In the nutrient-rich medium, single-use consumption of metabolites predominated, and 
175 metabolites in the base and minimal media had more diverse metabolite use profiles with an

176 increasing representation of cross-fed, single-produced and co-produced metabolites (Fig. 3).

178 We then investigated the identity of metabolites in the different metabolite use patterns.

179 Metabolite groups with the highest number of co-consumed metabolites were amino acids and B

180 vitamins (Fig. 4, Table S4A-C). Tyrosine, tryptophan, proline, phenylalanine, glutamine,

181 asparagine and arginine were the most frequently co-consumed amino acids and biotin (B7) the

182 most co-consumed B vitamin. However, some B vitamins and cofactors were cross-fed, notably

183 thiamin (B1), pyridoxine 5-phosphate (B6), nicotinamide D-ribonucleotide, riboflavin (B2),

184 tetrahydrofolate (B9) and coenzyme A (Fig. 4). Among the carbon compounds, only glucose and

185 glycerol were consistently co-consumed in all three media. Other carbon compounds displayed

186 more diverse use profiles that differed for each growth medium. For instance, malate and

187 formate were exclusively produced in the nutrient-rich and base media and consumed or cross-

188 fed in the minimal medium (Fig. 4); while lactate and 2,3 butanediol were exclusively produced

189 in the nutrient-rich medium. Intermediates of the TCA cycle 2-oxoglutarate, succinate, succinyl-

190 CoA, and fermentation products acetate, acetaldehyde and acetoin were cross-fed carbon at high

191 frequency; and serine, glutamate, and glycine were the most cross-fed amino acids (Fig. 4).

192 Among the nucleotides, the pyrimidine deoxyuridine 5'-phosphate (dUMP) simultaneously

193 ranked as the most co-consumed and cross-fed nucleotide (Fig. 4).

195 Metabolic roles of individual bacteria. Our next analyses focused on metabolite production

196 and consumption profiles of individual gut bacteria. Our simulations show that the metabolic

197 role of individual gut bacteria as source or sink varies with the identity of co-culture microbe and 
across the three media for many metabolites, but is generally conserved across communities

199 within the same media type (Fig. 5, Table S4D). For instance, all five gut bacteria produced

200 ammonia in the nutrient-rich and base media but consumed ammonia in the minimal medium

201 (Fig. 5A). . In base and rich media most microbes, except $A$. tropicalis, displayed committed

202 roles for the production or consumption of all metabolites (Table S5A-C); A. tropicalis alternated

203 roles as a producer and consumer for up 6\% of all metabolites (Table S5A-C). In the minimal

204 medium, all three acetic acid bacteria displayed variable roles as producers and consumers for up

205 to $\sim 30 \%$ of all metabolites (Table S5A-C). As an example, representatives of Acetobacter

206 alternated as sources and sinks for acetate, arginine, ornithine, and succinyl-CoA in the minimal

207 medium, depending on the number and identity of co-occurring bacteria (Fig.5B).

209 Furthermore, the bacterial taxa had distinctive metabolic characteristics. All three Acetobacter

210 species were sinks for glycine, serine, proline, acetaldehyde, methylglyoxal and producers of D-

211 alanine, cysteine, histidine, isoleucine, leucine, valine, formaldehyde, malate, succinate (Fig.

212 5A), and A. tropicalis was, additionally, an important source of B vitamins including

213 tetrahydrofolate (B9), riboflavin (B2), pyridoxine 5-phosphate (B6) and biotin (B7) (Fig. 5B).

214 Both Lactobacillus species produced acetoin and were sinks for tryptophan and the branched-

215 chain amino acids isoleucine, leucine, and valine. L. brevis was consistently a source of

216 succinate, deoxyuridine 5'-phosphate (dUMP) and sink for most amino acids and meso-2,6-

217 diaminoheptanediote (required for peptidoglycan synthesis). L. plantarum was a sink for

218 arginine and succinate and source of pyruvate and meso-2,6-diaminoheptanediote. 
Effect of community size and taxa on metabolite richness. We next considered how the

221 number of metabolites consumed or released by individual bacteria (i.e. metabolite richness) was

222 influenced by the number and identity of other bacteria in the community. For the nutrient-rich

223 and basal media, the number of taxa in a community did not significantly influence the number

224 of metabolites consumed or released by individual taxa (Fig. 6A, Table S4D). However, on the

225 minimal medium, as the number of taxa in a community increased, the number of metabolites

226 consumed and released also increased (Fig. 6A).

228 The metabolic function of individual microbial taxa significantly varied across all media types

229 (Table S4D). In particular, both Lactobacillus species consumed more metabolites than the three

230 Acetobacter species. A. tropicalis released more metabolites on average than the other two

231 Acetobacter species, and both Lactobacillus species released slightly more metabolites on

232 average than the grand mean (Fig. 6B).

233

234 The types of metabolites consumed or released varied with the identity and number of species in

235 the community (Fig. 6C, Table S5D). A. tropicalis and L. brevis had the strongest effect on

236 consumption rates, especially in the nutrient-rich medium; A. tropicalis positively correlated

237 with consumption of sulfur-containing metabolites, while L. brevis increased with vitamin

238 consumption (Fig. 6C). For metabolite release, Lactobacillus species had the greatest impact in

239 the nutrient-rich medium, correlated with vitamin and nucleotide release, while the Acetobacter

240 species were more important in the minimal depleted medium and A. tropicalis, in particular,

241 was strongly correlated with the release of sulfur-containing metabolites (Fig. 6C). A. pomorum

242 consistently had no effect on consumption or production rates (Table S5D). 
244 Net outputs from the bacterial communities. Our final analysis addressed the metabolic

245 products of the 31 bacterial communities in each of the three media. These products are

246 candidate bacterial-derived metabolites that are available to the host animal. A total of 21

247 metabolites were predicted to be made available to the host (Table S6, Dataset S2), 19 of which

248 were produced in the nutrient-rich medium, 12 in the base medium and five in the minimal

249 medium. Some of the 21 metabolites have been demonstrated to previously play important roles

250 in Drosophila physiology. For instance, acetate $(26,42,43)$ and succinate $(44)$ reduce host TAG

251 levels, and microbe-derived amino acids rescue Drosophila growth on amino acid deficient diets

$252(38,45)$ and double Drosophila lifespan on low protein diets (46).

253 Three metabolites (acetate, D-alanine, homocysteine) were predicted to be made available to the

254 host under all three diet conditions. In addition, the central carbon metabolites predicted to be

255 released from bacteria in at least one medium include 2-oxoglutarate, formaldehyde, formate,

256 glycolate, lactate, malate, and succinate.

\section{DISCUSSION}

259 Our in silico study of metabolic interactions among Drosophila gut bacteria yielded two key

260 findings. First, the pattern of metabolite consumption and release by individual bacteria and

261 communities is dynamic, varying with nutrient conditions and community composition. Second,

262 ecological interactions identified from the growth patterns of the bacteria (competition,

263 mutualism, etc) are underpinned by a diversity of metabolic interactions, with evidence that the

264 bacteria tend to compete for certain classes of nutrients (e.g. amino acids, B vitamins) more

265 frequently than for others, particularly carbon sources. 
267 Our modeling is based on several simplifying assumptions. In particular, we assume that the

268 different bacterial species are in close proximity, such that among-species flux of metabolites is

269 unimpeded. Although there is very limited information on the spatial organization of gut

270 microorganisms in Drosophila, bacteria in other hosts can be planktonic in the gut lumen or

271 adhere to the gut wall, often as single-species or structured multi-species colonies (47-50); and

272 these different spatial patterns both affect and are influenced by abiotic conditions, nutrient

273 availability, metabolite exchange and types of growth interactions between species (51-53). For

274 example, commensal and neutral interactions which are likely to occur among spatially isolated

275 microbes are not captured in most of our simulations. Furthermore, our models address primary

276 metabolism exclusively and are not designed to investigate the effects of secondary metabolite

277 mediated interactions, e.g. interference competition among bacteria mediated by toxins $(54,55)$,

278 differential susceptibility of bacteria to host immune factors (56-59). Despite these limitations,

279 many of our model outputs are consistent with published empirical data on the metabolic

280 function of Drosophila gut bacteria, particularly the production by individual bacterial species of

281 specific fermentation products $(24,60)$, amino acids $(30,61,62)$ and B vitamins $(29,63,64)$.

282 Furthermore, the predicted incidence of different ecological interactions, as deduced from

283 biomass production in the various communities, agrees largely with published data reporting a

284 predominance of antagonistic interactions among Drosophila-associated gut microbes (65) and

285 other microbial communities $(55,66-68)$. Our observation that nutrient-poor conditions favor

286 mutualistic interactions, especially in more complex communities, is also consistent with both

287 predictions and empirical data for other microbial systems, e.g. (69-71). Taken together, these

288 considerations indicate that our modeling approach is robust. It can be used with confidence to 
investigate patterns in the metabolic consequences of varying nutrient availability and

290 community composition over a larger range of conditions than is technically realistic for

291 empirical study.

292

293 Genome-scale metabolic modeling, as used here, brings into sharp focus the complexity of

294 metabolic interactions among microorganisms. This provides a different perspective from

295 empirical studies that, generally, focus on a single class of nutrients, e.g. short-chain fatty acids

296 or B vitamins. In particular, the metabolic traits of an individual bacterium are not fixed but

297 strongly influenced by the nutrient environment and the presence and identity of co-occurring

298 microorganisms. For example, our models predict that the Lactobacillus species are net

299 producers of uracil only under nutrient-rich conditions (Fig. 5). Bacterial-derived uracil has been

300 shown to induce a pro-inflammatory state in the Drosophila gut via DUOX-mediated production

301 of reactive oxygen species (72), and our data raise the possibility that the effect of Lactobacillus

302 on the immunological status of the gut may be influenced by dietary factors. Similarly, the

303 finding that the net production of several B vitamins by Acetobacter varies with the presence and

304 identity of co-occurring bacteria (Fig. 5A) suggests that studies exclusively using associations

305 with single bacterial taxa may not capture the full complexity of B vitamin provisioning by the

306 Drosophila gut microbiome.

307

308 The complexity of the metabolic interactions among the bacterial species also has implications

309 for the sign of ecological interactions. Various empirical analyses have demonstrated how an

310 ecological interaction can be driven by a single metabolic interaction, e.g. competition for a

311 single resource (73-75), mutualism by reciprocal cross-feeding of a pair of metabolites, each 
312 produced by one microorganism and required by the other $(76,77)$. However, as summarized in

313 Fig. 3, the totality of the metabolic relationship between interacting microorganisms includes

314 multiple classes of metabolic interaction. A relatively minor change in the uptake/release of

315 metabolite(s), in response to a change in nutrient availability or community composition, could

316 result in the transition to a different ecological relationship. For example, the switch from a

317 competitive interaction to parasitism may contribute to the bloom of a microorganism previously

318 held in check by competition (78).

320 Further elaboration of metabolic models, as used here and, for example by (68), offers the

321 opportunity to investigate how subtle changes in metabolite flux in communities of different

322 complexity and different nutrient regimes can lead to a switch between different ecological states

323 of the microbiome and its interaction with the host.

325 The complexity and variation in metabolic interactions among the gut bacteria are, however,

326 overlain by several broad patterns with respect to both metabolite class and bacterial species.

327 Considering metabolites first, a key output of this study is that the pattern of metabolite use

328 differed between substitutable and non-substitutable nutrient sources, i.e. where other resources

329 can be utilized as an equivalent resource versus where no equivalent resource is available (79).

330 Consistent with the generality that organisms tend to compete less for substitutable than non-

331 substitutable resources (80), the Drosophila gut bacteria competed more for non-substitutable

332 nutrients, such as amino acids and B vitamins, than substitutable nutrients, such as intermediates

333 in central carbon metabolism. These patterns have implications for the health and wellbeing of

334 the host. In particular, microbial consumption of dietary nutrients can deplete nutrient 
335 availability to the host (81), and among-microbe competition is predicted to alter host access to

336 both critical nutrients and metabolites that influence the signaling pathways in Drosophila (26,

337 82) and other animals $(83,84)$.

339 The five bacteria selected for this analysis varied in their genetic capacity for metabolic function,

340 particularly between the Acetobacter and Lactobacillus species (Fig. 6, see also (39)).

341 Nevertheless, our modeling revealed substantial functional redundancy in metabolite production

342 among the different bacteria: except for meso-2,6-diaminoheptanediote, produced by $L$.

343 plantarum, we identified no metabolites produced exclusively by a single species. This

344 metabolic redundancy is consistent with the evidence that taxonomically-different microbial

345 communities can be functionally equivalent in Drosophila (85) and other animals $(86,87)$. The

346 unique metabolic function of L. plantarum highlights the role of this bacterium in microbial

347 community interactions, including its potential as a probiotic. The beneficial effects of $L$.

348 plantarum in the Drosophila system have been attributed to its capacity to promote protein

349 assimilation from the diet (82). Our observation that L. plantarum provisions cell wall

350 constituents, B vitamins and amino acids for auxotrophic bacteria in the gut provides additional

351 metabolic routes by which L. plantarum may promote the overall diversity of the gut microbiota.

353 In conclusion, this study demonstrates how in silico approaches can yield mechanistic insight

354 into the metabolic traits of individual microbes and communities, and how these traits can

355 influence metabolite levels that impact host physiology. Our models identify patterns by which

356 microbial communities interact and respond to changes in nutrient input from the host and allow

357 the generation of testable hypotheses for more targeted empirical studies. Substantial insight into 
358 how variations in microbiomes impact host health and metabolism have been gained from

359 combining metabolic modeling with empirical studies $(88,89)$ and the simplicity of the

360 Drosophila system presents an ideal model system to combine in silico and in vivo approaches to

361 understand how gut-associated microbes impact host health.

362

363 MATERIALS AND METHODS

364

365 Generation of the individual bacterial metabolic models. Genomes of Acetobacter fabarum

366 (JOPD01000000), Acetobacter pomorum (JOKL01000000), Acetobacter tropicalis

367 (JOKM01000000), Lactobacillus brevis (JOKA01000000) and Lactobacillus plantarum

368 (JOJT01000000) were downloaded from NCBI and re-annotated using the RAST annotation

369 server (90). Two draft model reconstructions were generated for each genome and combined to

370 generate a final model. The first models were obtained by performing reciprocal BLASTs of

371 Acetobacter genomes against Escherichia coli str. K-12 substr. MG1655 and Lactobacilli

372 genomes against Lactobacillus plantarum WCFS1. Gene orthologs identified from the

373 reciprocal blast searches were compared to the E. coli str. K-12 substr. MG1655 metabolic

374 model iML1515 (91) and the Lactobacillus plantarum WCFS1 metabolic model (92). Then,

375 reactions encoded by these genes were manually extracted to create a draft model. The second

376 draft reconstructions were generated from the automated reconstruction pipeline ModelSEED

377 (93) using the RAST re-annotated genomes as input. For each bacterium, the two models were

378 integrated and manually curated to remove redundant reactions and ensure correct reaction gene

379 association, stoichiometry, and directionality. Organism-specific features and genes encoding

380 metabolic reactions absent in Escherichia coli iML1515 and Lactobacillus plantarum WCFS1 
metabolic models were identified by literature review and searches of BioCyc, KEGG, EcoCyc, BiGG and BRENDA databases (94-98), and then added to the draft model. The models were

383 further curated using nutrient utilization BioLog data from (39) to verify and identify nutrient

384 sources utilized by Acetobacter and Lactobacillus. All models were evaluated using MEMOTE (99).

Model media composition. All simulations were performed in one of three media types; a minimal medium, a base medium and a rich medium. The minimal medium is a nutrient poor medium containing glucose, glycerol, ammonia, sulfate, and phosphate as primary sources of carbon, nitrogen, sulfur, and phosphorus, respectively. Components of the minimal medium

391 were selected to investigate the complete scope of interaction possible between Drosophila gut

392 microbiota in the absence of host gut and diet derived nutrients. Components of the base

393 medium were selected to match nutrient auxotrophies for all 5 bacteria and allow the growth of

394 all 5 bacteria in isolation. Components of the rich medium comprised the complete set of

395 nutrients required for growth by all five bacteria, all major sources of carbon, nitrogen, sulfur,

396 and phosphorus that all the bacteria have annotated transporters for, nutrient components of

397 Drosophila holidic diet and metabolites predicted to be available in the fly gut from a

398 metabolomic analysis (100). All media nutrient constituents and the flux bounds used for all

399 bacteria and all simulations are provided in Table S7.

401 Model constraints applied. Reaction fluxes for community members were obtained using the 402 SteadyCom (41) Flux Variability Analysis (FVA) implementation in the OpenCOBRA Toolbox 403 (101) with the following constraints. Medoid growth rate vectors of individual species were 
404 computed by performing FVA while maintaining $99.99 \%$ of the maximum community growth

405 rate $(\mu \max )$ and simultaneously maximizing and minimizing flux through individual reactions to

406 obtain individual species growth rate values for each simulation. For each species, we obtained

407 between 1000-5000 growth rate values, representing species growth rates when each reaction

408 was performing at its minimum and maximum while maintaining the maximum community

409 growth rate. We used the medoid predicted growth rate value for each species as a lower bound

410 for all subsequent model simulations by constraining SteadyCom parameters BMcon, BMrhs,

411 and BMCsense to require each species have a biomass value of at least the computed medoid in

412 all simulations. A SteadyCom algorithm that minimizes the L1 (taxicab) norm of the predicted

413 flux vectors was also applied to remove futile cycles and extraneous flux predictions.

415 Model simulation. To find the maximum community growth rate we used the bisection

416 algorithm in SteadyCom because it presented minimal convergence and feasibility issues with

417 the constraints applied to our community model. Additionally, the default SteadyCom feasTol

418 algorithm which sets the allowed error for determining if an input solution is feasible was set to

419 1e-8 for the solver. All parameters used to constrain SteadyCom simulations are available in the 420 runSteadyCom and runSteadyComFVAMedoid functions.

422 Analysis of Simulated Results. Simulations were run for each subset of the 5 species, i.e. $2^{5}-1=$

42331 communities, 5 of which are single-species communities. We constructed the single-member

424 communities as multi-species models to facilitate the analysis and simulation pipelines,

425 particularly SteadyCom, which requires a multi-species model. We verified that growth rates for 
426 single-species models and the single-member community models with flux balance analysis

427 (102) were identical (see Tests/testMultiModelSingleton.m).

429 Statistical analyses. All statistical analyses were performed using R v.3.6.1 (103) with an alpha

430 of 0.05 to assess significance. Bonferroni correction for multiple tests was applied where

431 required. Statistical differences between the number of overlapping metabolites in different

432 community sizes and metabolite use patterns associated with different ecological interactions

433 were investigated by one-way analysis of variance (ANOVA) followed by Tukey's HSD post hoc

434 test. Metabolite richness was calculated as the number of metabolites either consumed or

435 released by a given taxon in each microbial treatment and medium combination. A mixed effect

436 two-way ANOVA was used to assess how the medium type (rich, basal, or minimal) and

437 community size (number of taxa) influenced metabolite richness using 'lmer' function in lme4

438 package (104). The data for metabolite consumption and release were analyzed separately.

439 Medium type and community size were included as categorical fixed effects and microbial

440 treatment (combination of microbes in a community, e.g. AF + LB) and taxon (AF, AP, AT, LB,

441 or LP) were designated categorical random effects. The 'Anova' function in the car package

442 (105) was used to perform a type III Wald's F test to determine the effect of predictors with a

443 Kenward-Rodger approximation to estimate residual degrees of freedom. A post hoc Tukey's

444 test was performed for each media type to determine pairwise differences across community size.

445 An analysis of deviance was performed to assess the significance of each random effect and the

446 best linear unbiased prediction was estimated for each taxon using the 'ranef' function to predict

447 the global effect of each taxon across the different community size and medium combinations.

448 Marginal and conditional R2 values were calculated using the MuMIn package (106). 
450 A principal component analysis (PCA) was performed to correlate metabolite consumption or

451 release rates with community size, medium type, and microbial presence using the vegan

452 package (107). A correlation matrix was implemented using the total sum rate of metabolites

453 either consumed or released for each of the metabolite type bins (carbon, amino acids, nitrogen,

454 etc.). The function 'envfit' was used to correlate PC1 and PC2 with microbial presence with 999

455 permutations and significant vectors were plotted. In addition, a permutational multivariate

456 analysis of variance (PERMANOVA) was used to assess the effect of community size by

457 medium type as well as taxon on metabolite rates with the function 'adonis'. Data was

458 autoscaled and a Euclidean distance matrix was implemented for the model with 999

459 permutations. Consumption and release rates were analyzed separately for all analyses.

461 Code and Data Availability. All code used in this study can be found at

462 https://github.com/federatedcloud/DouglasMetabolicModels/releases/tag/v1.0.1. The

463 simulations were performed using v3.0.4 of the OpenCOBRA Toolbox and v7.5.1 of the Gurobi

464 Optimizer (108). An optional, containerized environment for running the code is available at

465 https://github.com/federatedcloud/COBRAContainers. All results derived from simulations can

466 be found in DouglasMetabolicModels/analysis/CMP_and_CooperativeFluxes. A tutorial is

467 provided for performing simulations in the repository's top-level README file. The code is

468 available under the MPL2 license. 
470 SBML files of the models have been submitted to the BioModels database (109) with the

471 following identifiers: MODEL2002040002, MODEL2002040003, MODEL2002040004,

472 MODEL2002040005 and MODEL2002040006.

473

474 ACKNOWLEDGEMENTS

475 We thank the Aristotle Cloud Federation (NSF OAC-1541215) for computing resources for 476 model simulation, and Dr. Lynn Johnson (Cornell Statistical Consulting Unit) for statistical

477 advice. This research was funded by an NIH grant R01GM095372 to AED.

479 There is no conflict of interest for all authors.

Author contributions. NYDA, BEB, and AED designed research; NYDA and JS created

482 metabolic models; NYDA, BEB and CW performed model simulations and analysis; JGM

483 performed statistical analysis; NYDA and AED wrote the first draft of the paper, and manuscript

484 revisions were made by all authors.

485

\section{REFERENCES}

1. Adair KL, Douglas AE. 2017. Making a microbiome: the many determinants of host-associated microbial community composition. Curr Opin Microbiol 35:23-29. application of ecological theory toward an understanding of the human microbiome. Science 336:1255-1262. 
3. Douglas AE. 2018. Fundamentals of Microbiome Science: How Microbes Shape Animal Biology. Princeton University Press. stability and resilience of the human gut microbiota. Nature 489:220-230. Douglas AE, Dubilier N, Eberl G, Fukami T, Gilbert SF, Hentschel U, King N, Kjelleberg S, Knoll AH, Kremer N, Mazmanian SK, Metcalf JL, Nealson K, Pierce NE, Rawls JF, Reid A, Ruby EG, Rumpho M, Sanders JG, Tautz D, Wernegreen JJ. 2013. Animals in a bacterial world, a new imperative for the life sciences. Proc Natl Acad Sci USA 110:3229-3236. beneficial microorganisms associated with insects. Environ Microbiol 20:2002-2011. PLoS Pathog 15: e1007727. Westmiller S, Wong AC-N, Clark AG, Lazzaro BP. 2015. Host genetic determinants of microbiota-dependent nutrition revealed by genome-wide analysis of Drosophila melanogaster. Nature Comm 6:6312. Spector TD, Bell JT, Clark AG, Ley RE. 2016. Genetic determinants of the gut microbiome in UK twins. Cell Host Microbe 19:731-743. microbiota. Nature Rev Gastroent Hepatol 16:35-56. 
12. Wymore Brand M, Wannemuehler MJ, Phillips GJ, Proctor A, Overstreet A-M, Jergens AE, Orcutt RP, Fox JG. 2015. The altered Schaedler flora: continued applications of a defined murine microbial community. ILAR J 56:169-178. microbial communities to model host-microbe interactions in the human gut. Microbiol Mol Biol Rev 83:e0054-18. A, Carlson JM, Beerenwinkel N, Ludington WB. 2018. Microbiome interactions shape host fitness. Proc Natl Acad Sci USA 115:E11951-E11960.

15. Ankrah NY, Chouaia B, Douglas AE. 2018. The cost of metabolic interactions in symbioses between insects and bacteria with reduced genomes. mBio 9:e01433-18. The microbiome modeling toolbox: from microbial interactions to personalized microbial communities. Bioinformatics 35:2332-2334. of the human gut microbiota. mSystems 3:e00209-17. human microbiome elucidates community-level assembly rules. Proc Natl Acad Sci USA 110:12804-12809. melanogaster. Gut Microbes 3:307-321. 
22. Chaston JM, Newell PD, Douglas AE. 2014. Metagenome-wide association of microbial determinants of host phenotype in Drosophila melanogaster. mBio 5:e0163114.

23. Cox CR, Gilmore MS. 2007. Native microbial colonization of Drosophila melanogaster and its use as a model of Enterococcus faecalis pathogenesis. Infect Immun $75: 1565-1576$.

24. Fischer C, Trautman EP, Crawford JM, Stabb EV, Handelsman J, Broderick NA. 2017. Metabolite exchange between microbiome members produces compounds that influence Drosophila behavior. eLife 6:e18855.

25. Newell PD, Douglas AE. 2014. Interspecies interactions determine the impact of the gut microbiota on nutrient allocation in Drosophila melanogaster. Appl Environ Microbiol 80:788-796. W-J. 2011. Drosophila microbiome modulates host developmental and metabolic homeostasis via insulin signaling. Science 334:670-674. plantarum promotes Drosophila systemic growth by modulating hormonal signals through TOR-dependent nutrient sensing. Cell Metab 14:403-414. Simpson SJ, Ponton F. 2017. Gut microbiota modifies olfactory-guided microbial preferences and foraging decisions in Drosophila. Curr Biol 27:2397-2404. e4. metabolic response of Drosophila to diet. J Exp Biol 217:1894-1901. growth. PLoS Biol 18:e3000681. 
31. Sommer AJ, Newell PD. 2019. Metabolic basis for mutualism between gut bacteria and its impact on the Drosophila melanogaster host. Appl Environ Microbiol 85:e01882-18.

32. Cleenwerck I, Gonzalez A, Camu N, Engelbeen K, De Vos P, De Vuyst L. 2008. Acetobacter fabarum sp. nov., an acetic acid bacterium from a Ghanaian cocoa bean heap fermentation. Int J Syst Evol Microbiol 58:2180-2185.

33. Zheng J, Ruan L, Sun M, Gänzle M. 2015. A genomic view of lactobacilli and pediococci demonstrates that phylogeny matches ecology and physiology. Appl Environ Microbiol 81:7233-7243.

34. Chaston JM, Dobson AJ, Newell PD, Douglas AE. 2016. Host genetic control of the microbiota mediates the Drosophila nutritional phenotype. Appl Environ Microbiol 82:671-679.

35. Wang Y, Kapun M, Waidele L, Kuenzel S, Bergland AO, Staubach F. 2020. Common structuring principles of the Drosophila melanogaster microbiome on a continental scale and between host and substrate. Environ Microbiol Rep 12:220-228.

36. Douglas AE. 2018. The Drosophila model for microbiome research. Lab Anim 47:157-164.

38. Consuegra J, Grenier T, Baa-Puyoulet P, Rahioui I, Akherraz H, Gervais H,

37. Adair KL, Wilson M, Bost A, Douglas AE. 2018. Microbial community assembly in wild populations of the fruit fly Drosophila melanogaster. ISME J 12:959-972. Parisot N, da Silva P, Charles H, Calevro F. 2020. Drosophila-associated bacteria differentially shape the nutritional requirements of their host during juvenile growth. PLoS Biol 18:e3000681. AJ, Kagle J, Douglas AE. 2014. In vivo function and comparative genomic analyses of the Drosophila gut microbiota identify candidate symbiosis factors. Front Microbiol 5:576. 
40. Sannino DR, Dobson AJ, Edwards K, Angert ER, Buchon N. 2018. The Drosophila melanogaster gut microbiota provisions thiamine to its host. mBio 9:e0015518. abundances while ensuring community stability. PLoS Comput Biol 13:e1005539.

42. McMullen JG, Peters-Schulze G, Cai J, Patterson AD, Douglas AE. 2020. How gut microbiome interactions affect nutritional traits of Drosophila melanogaster. $J$ Exp Biol 223: jeb227843. Drosophila immune deficiency pathway modulates enteroendocrine function and host metabolism. Cell Metab 28:449-462.e5. microbial product that modulates Drosophila nutritional physiology. Insect Science. 2020. Metabolic cooperation among commensal bacteria supports Drosophila juvenile growth under nutritional stress. iScience 23:101232.

46. Leitão-Gonçalves R, Carvalho-Santos Z, Francisco AP, Fioreze GT, Anjos M, Baltazar C, Elias AP, Itskov PM, Piper MD, Ribeiro C. 2017. Commensal bacteria and essential amino acids control food choice behavior and reproduction. PLoS Biol 15:e2000862. PJ. 2012. A crypt-specific core microbiota resides in the mouse colon. mBio 3:e00116-12.

48. Yasuda K, Oh K, Ren B, Tickle TL, Franzosa EA, Wachtman LM, Miller AD, Westmoreland SV, Mansfield KG, Vallender EJ, Miller GM, Rowlett JK, Gevers D, Huttenhower C, Morgan XC. 2015. Biogeography of the intestinal mucosal and lumenal microbiome in the rhesus macaque. Cell Host Microbe 17:385-391. 
49. Mark Welch JL, Hasegawa Y, McNulty NP, Gordon JI, Borisy GG. 2017. Spatial organization of a model 15-member human gut microbiota established in gnotobiotic mice. Proc Natl Acad Sci USA 114:E9105-E9114.

50. Schlomann BH, Wiles TJ, Wall ES, Guillemin K, Parthasarathy R. 2018. Bacterial Cohesion Predicts Spatial Distribution in the Larval Zebrafish Intestine. Biophys J 115:2271-2277.

51. Borer B, Tecon R, Or D. 2018. Spatial organization of bacterial populations in response to oxygen and carbon counter-gradients in pore networks. Nat Commun 9:769. 2017. Spatial organization plasticity as an adaptive driver of surface microbial communities. Front Microbiol 8:1364. competition in biofilms. Nat Rev Microbiol 14:589-600. of Bacterial Warfare. Curr Biol 29:R521-R537. surviving and thriving in the microbial jungle. Nat Rev Microbiol 8:15-25. macrophages in shaping gut microbiota in adult zebrafish. Cell Rep 25:424-436. antimicrobial peptides in gut immunity and Drosophila-microbe interactions. Phil Trans R Soc Lond B Biol Sci 371:20150295. antibacterial, and antiviral mechanisms in the gut. PLoS Pathog 13:e1006277. 
59. Pabst O, Slack E. 2020. IgA and the intestinal microbiota: the importance of being specific. Mucosal Immunol 13:12-21.

60. Kim G, Huang J, McMullen II JG, Newell PD, Douglas AE. 2017. Physiological responses of insects to microbial fermentation products: insights from the interactions between Drosophila and acetic acid. J Insect Physiol 106:13-19.

61. Leitao-Goncalves R, Carvalho-Santos Z, Francisco AP, Fioreze GT, Anjos M, Baltazar C, Elias AP, Itskov PM, Piper MDW, Ribeiro C. 2017. Commensal bacteria and essential amino acids control food choice behavior and reproduction. PLoS Biol 15:e2000862.

62. Judd AM, Matthews MK, Hughes R, Veloz M, Sexton CE, Chaston JM. 2018. Bacterial methionine metabolism genes influence Drosophila melanogaster starvation resistance. Appl Environ Microbiol 84:e00662-18. symbiotic folate production on fitness and development in the fruit fly Drosophila melanogaster. Fly 4:312-319. Drosophila melanogaster gut microbiota provisions thiamine to its host. mBio 9:e0015518. A, Carlson JM, Beerenwinkel N, Ludington WB. 2018. Microbiome interactions shape host fitness. Proc Natl Acad Sci USA 115:E11951-E11960. among culturable microbial species. Curr Biol 22:1845-50. Widder S. 2018. Signatures of ecological processes in microbial community time series. Microbiome 6:120. 
68. Venturelli OS, Carr AC, Fisher G, Hsu RH, Lau R, Bowen BP, Hromada S, Northen T, Arkin AP. 2018. Deciphering microbial interactions in synthetic human gut microbiome communities. Mol Syst Biol 14:e8157.

69. Zelezniak A, Andrejev S, Ponomarova O, Mende DR, Bork P, Patil KR. 2015. Metabolic dependencies drive species co-occurrence in diverse microbial communities. Proc Natl Acad Sci USA 112:6449-54. Sharan R, Ruppin E. 2011. Competitive and cooperative metabolic interactions in bacterial communities. Nat Commun 2:589. availability modulates the cooperative and competitive nature of a microbial crossfeeding mutualism. PLoS Biol 14:e1002540. Lee WJ. 2013. Bacterial-derived uracil as a modulator of mucosal immunity and gutmicrobe homeostasis in Drosophila. Cell 153:797-811. experimental populations of yeast. Nature 441:498-501.

74. Vulic M, Kolter R. 2001. Evolutionary cheating in Escherichia coli stationary phase cultures. Genetics 158:519-26. J, Friedman J, Blainey PC. 2019. Massively parallel screening of synthetic microbial communities. Proc Natl Acad Sci USA 116:12804-12809. 
77. D'Souza G, Shitut S, Preussger D, Yousif G, Waschina S, Kost C. 2018. Ecology and evolution of metabolic cross-feeding interactions in bacteria. Nat Prod Rep 35:455488.

78. Selber-Hnatiw S, Rukundo B, Ahmadi M, Akoubi H, Al-Bizri H, Aliu AF, Ambeaghen TU, Avetisyan L, Bahar I, Baird A, Begum F, Ben Soussan H, BlondeauEthier V, Bordaries R, Bramwell H, Briggs A, Bui R, Carnevale M, Chancharoen M, Chevassus T, Choi JH, Coulombe K, Couvrette F, D'Abreau S, Davies M, Desbiens MP, Di Maulo T, Di Paolo SA, Do Ponte S, Dos Santos Ribeiro P, Dubuc-Kanary LA, Duncan PK, Dupuis F, El-Nounou S, Eyangos CN, Ferguson NK, Flores-Chinchilla NR, Fotakis T, Gado Oumarou HDM, Georgiev M, Ghiassy S, Glibetic N, Gregoire Bouchard et al. 2017. Human gut microbiota: toward an ecology of disease. Front Microbiol 8:1265.

79. Tilman D. 1982. Resource Competition and Community Structure. Princeton University Press, Princeton, NJ. complementary or substitutable resources. J Theo Biol 50:185-201. determines Drosophila lipid content. Biol Lett 11:20150469. plantarum promotes Drosophila systemic growth by modulating hormonal signals through TOR-dependent nutrient sensing. Cell Metab 14:403-14. microbiota and the immune system. Genes Dev 30:1589-97. Nutr 57:1-24. 
85. Kang D, Douglas AE. 2020. Functional traits of the gut microbiome correlated with host lipid content in a natural population of Drosophila melanogaster. Biol Lett 16:20190803.

86. Lozupone CA, Stombaugh JI, Gordon JI, Jansson JK, Knight R. 2012. Diversity, stability and resilience of the human gut microbiota. Nature 489:220-30. microbiota subjected to disturbance. Trends Microbiol 24:402-413. Systematic assessment of secondary bile acid metabolism in gut microbes reveals distinct metabolic capabilities in inflammatory bowel disease. Microbiome 7:75. fibrosis airway communities predicts mechanisms of pathogen dominance. mSystems 4:e00026-19.

90. Aziz RK, Bartels D, Best AA, DeJongh M, Disz T, Edwards RA, Formsma K, subsystems technology. BMC Genomics 9:75. Zhang Z, Mori H. 2017. iML1515, a knowledgebase that computes Escherichia coli traits. Nature Biotech 35:904. models. Nature Biotech 28:977-982. 
94. Caspi R, Foerster H, Fulcher CA, Kaipa P, Krummenacker M, Latendresse M, Paley S, Rhee SY, Shearer AG, Tissier C. 2008. The MetaCyc Database of metabolic pathways and enzymes and the BioCyc collection of Pathway/Genome Databases. Nuc Acids Res 36:D623-D631.

95. Kanehisa M, Goto S. 2000. KEGG: kyoto encyclopedia of genes and genomes. Nuc Acids Res 28:27-30.

96. Keseler IM, Mackie A, Peralta-Gil M, Santos-Zavaleta A, Gama-Castro S, Bonavides-Martínez C, Fulcher C, Huerta AM, Kothari A, Krummenacker M. 2013. EcoCyc: fusing model organism databases with systems biology. Nuc Acids Res 41:D605-D612.

97. Schellenberger J, Park JO, Conrad TM, Palsson BØ. 2010. BiGG: a Biochemical Genetic and Genomic knowledgebase of large scale metabolic reconstructions. BMC Bioinformatics 11:213.

98. Schomburg I, Chang A, Ebeling C, Gremse M, Heldt C, Huhn G, Schomburg D. 2004. BRENDA, the enzyme database: updates and major new developments. Nuc Acids Res 32:D431-D433.

99. Lieven C, Beber ME, Olivier BG, Bergmann FT, Ataman M, Babaei P, Bartell JA, Blank LM, Chauhan S, Correia K. 2020. MEMOTE for standardized genome-scale metabolic model testing. Nat Biotechnol 38:272-276. melanogaster nutrition. PhD Thesis. Cornell University. Haraldsdottir HS, Wachowiak J, Keating SM, Vlasov V. 2019. Creation and analysis of biochemical constraint-based models using the COBRA Toolbox v. 3.0. Nature Protoc 14:639. Biotech 28:245-248. 
103. R Core Team. 2019. R: A Language and Environment for Statistical Computing,

R Foundation for Statistical Computing, Vienna, Austria. https://www.R-project.org. Models Using lme4. JStatist Software 67:1-48. Sage, Thousand Oaks CA.

106. Bartoń K. 2019. MuMIn: Multi-Model Inference, vR package version 1.43.6. https://CRAN.R-project.org/package=MuMIn

Fig. 1 | Bacterial growth dynamics on media of different nutrient content. Growth dynamics

797 displayed as biomass formation rate predicted for growth in (A) nutrient rich medium, (B) base

798 medium, and (C) minimal medium. Bar colors represents specific bacteria.

800 of co-culture and medium on the sign of interactions between bacteria in the three test media... 
801 No commensal interactions were observed in any media type. (B) Overlapping metabolites

802 consumed by bacteria in the 31 simulated communities. Symbol colors correspond to individual

803 bacteria and the line connects the median value for the communities of different complexity.

804 Significantly different $(\mathrm{P}<0.05)$ groups by Tukey's HSD posthoc test are indicated by different 805 letters.

806 Fig. 3 | Metabolite use patterns associated with competitive, parasitic and mutualistic

807 growth outcomes. Each dot represents the frequency of a metabolite use pattern associated

808 between bacterial pairs in the 31 simulated communities. Black bar indicates median frequency

809 of occurrence for each metabolite use pattern. Significantly different $(\mathrm{P}<0.05)$ groups by

810 Tukey's HSD posthoc test are indicated by different letters.

811 Fig. 4 | Metabolite use patterns for metabolite classes amino acid, carbon, nucleotide,

812 vitamin and cofactors. Tick marks on $\mathrm{X}$-axis indicate relative frequency of the consumption or

813 production of a metabolite and range from 0 to 1 at 0.25 increments. The relative frequency of

814 metabolite use is calculated by dividing the number of times a metabolite is used in a particular

815 pattern (single-use, co-consumed, cross-fed, single-produced, co-produced) by the total number

816 of times the metabolite is produced or consumed in the 31 simulated communities.

817 Fig. 5 | Metabolic roles of individual bacteria. Predicted metabolite production and

818 consumption profiles for metabolite classes (A) nucleotide, vitamin and cofactors and (B) amino

819 acid and carbon. Two letter abbreviations at the top of each plot represent individual bacteria:

820 AF-Acetobacter fabarum, AP-Acetobacter pomorum, AT-Acetobacter tropicalis, LB-

821 Lactobacillus brevis, LP-Lactobacillus plantarum. Tick marks on X-axis indicate relative

822 frequency of the consumption or production of a metabolite and range from 0 to 1 at 0.25

823 increments. 


\section{Fig. 6 | Metabolic function of simulated microbial taxa under different conditions. (A)}

825 Effect of community size on metabolite richness. Metabolite richness is calculated as the number

826 of metabolites either consumed or released by a given taxon in each microbial treatment and

827 medium combination. Effect of community size for each medium is indicated with the estimated

828 marginal mean (open circles or diamonds) and standard error (SE) from ANOVA models. Letters

829 indicate results from post hoc Tukey's test, which was conducted separately for each medium.

830 Closed, colored circles indicate individual metabolite richness values for each taxon under each

831 condition. Effect test results are displayed in Table S4D. (B) Global effect of species identity on

832 metabolite richness for the number of compounds consumed and released. The conditional mean

833 and standard deviation are displayed for the best linear unbiased prediction. Dotted line indicates

834 the grand mean for metabolite richness across all species. (C) Principal component analysis

835 (PCA) correlating metabolite consumption or release rates with community size, medium type,

836 and microbial presence. Black arrows indicate metabolite type scores, and colored arrow display

837 the correlation vectors for microbial presence (only significant vectors are plotted). The percent

838 variance explained by each axis is shown in parentheses.

840 Supplementary Tables

841 Table S1. Predicted changes to bacterial growth (gdw h-1) in co-culture compared to growth in

842 monoculture A) rich medium, B) base medium, C) minimal medium.

843 Table S2. Predicted number of inputs and outputs from bacteria A) rich medium, B) base

844 medium, C) minimal medium. D) Summary statistics for Figure 2B 
845 Table S3. Predicted community metabolite use patterns for competitive, parasitic and mutualistic

846 interactions A) rich medium, B) base medium, C) minimal medium. D) Summary statistics for

847 Figure 3.

848 Table S4. Metabolite use pattern A) rich medium, B) base medium, C) minimal medium. D)

849 Effect of community size and medium type on metabolite richness. Tests with significant $\mathrm{p}$

850 values are shown in bold.

851 Table S5. Predicted total number of times metabolite is consumed or produced by individual

852 bacteria in all simulations A) rich medium, B) base medium, C) minimal medium. D) Effect of

853 taxa, community size, and medium type on metabolite consumption and release rates. Tests with

854 significant $\mathrm{p}$ values are shown in bold.

855 Table S6. Metabolites predicted to be available to the host.

856 Table S7. List of components A) rich medium, B) base medium, C) minimal medium.

858 Datasets

859 Dataset S1. A) Metabolite abbreviation key, B) Predicted number of inputs and outputs from

860 bacteria - rich medium, C) Predicted number of inputs and outputs from bacteria - base medium,

861 D) Predicted number of inputs and outputs from bacteria - minimal medium.

862 Dataset S2. Predicted metabolite flux available to to the host. 
Fig. 1
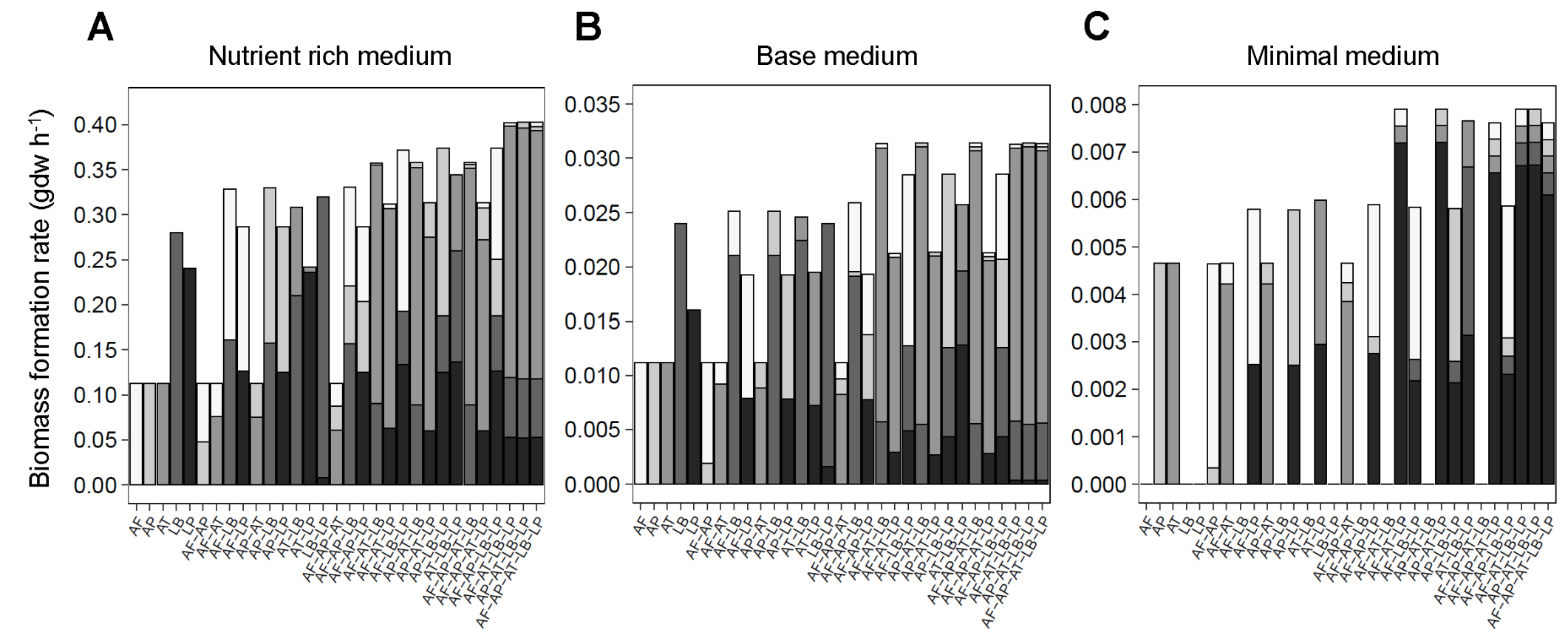

Community composition

A. fabarum (AF) $\bigcirc$ A. pomorum (AP) $\bigcirc$ A. tropicalis (AT) $\bigcirc$ L. brevis (LB) $\bigcirc$ L. plantarum (LP) 
Fig. 2

A

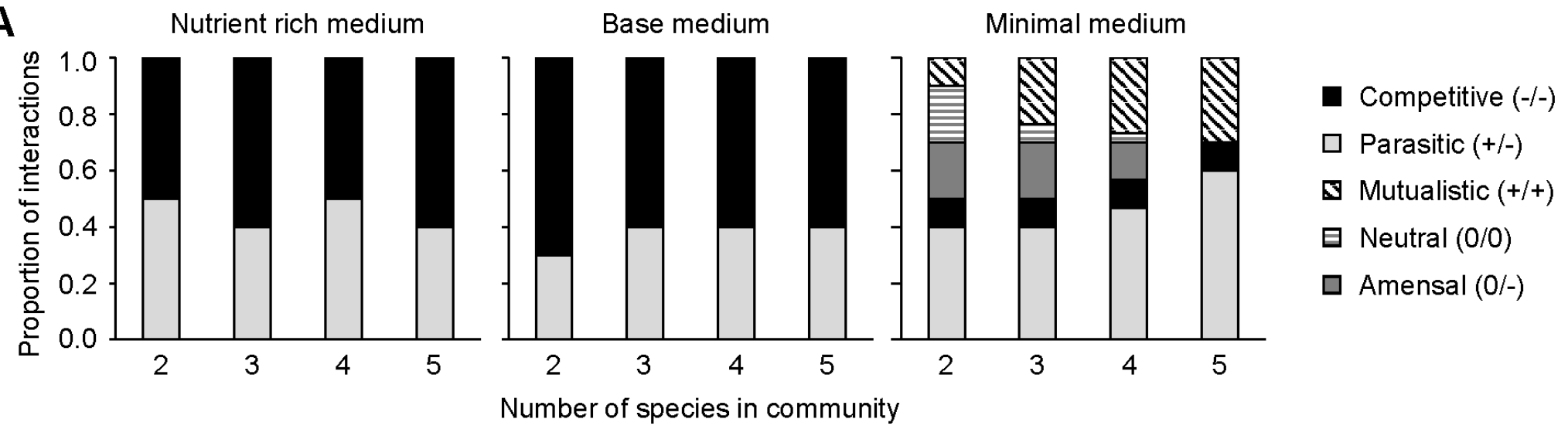

B

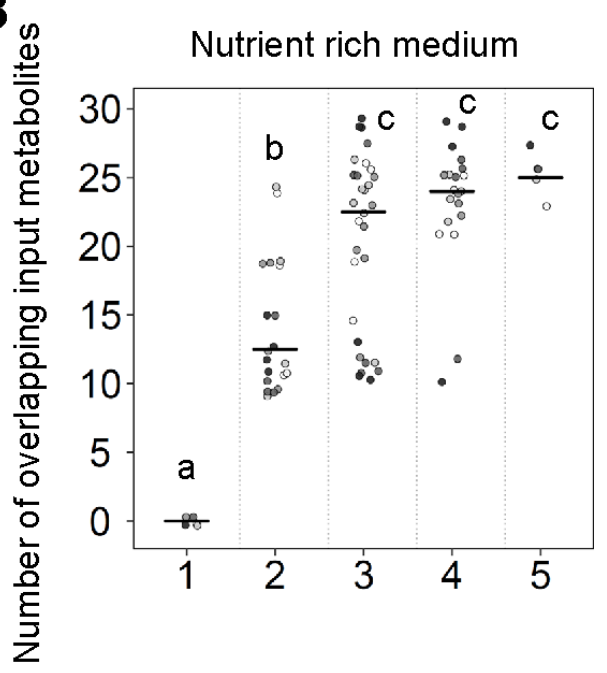

Base medium

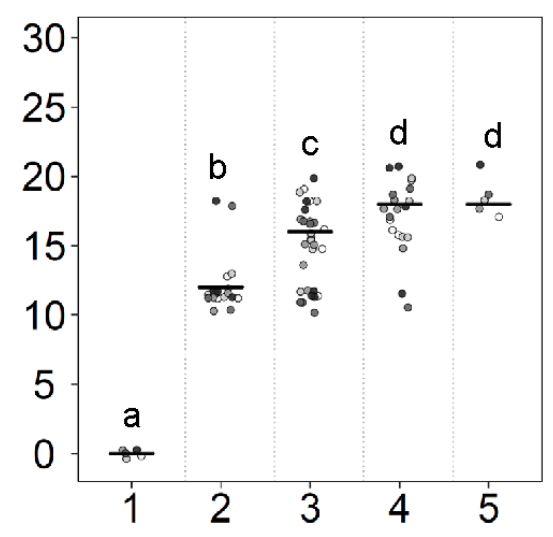

Minimal medium

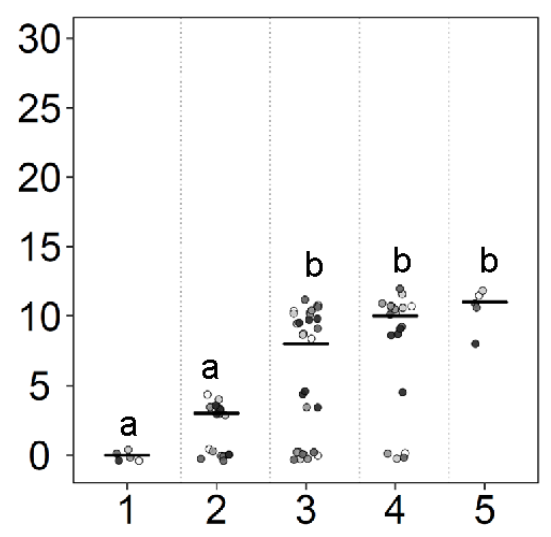

A. fabarum (AF)

A. pomorum (AP)

- A. tropicalis (AT)

L. brevis (LB)

- L. plantarum (LP) 


\section{Fig. 3}

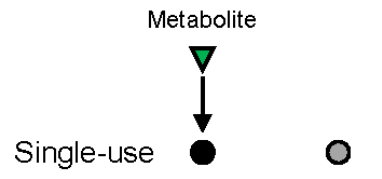

Microbe A Microbe B

Co-consumed

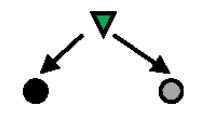

Cross-fed

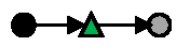

Single-produced
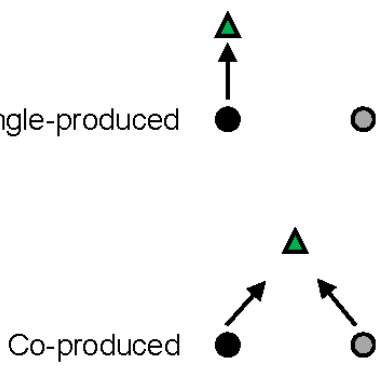

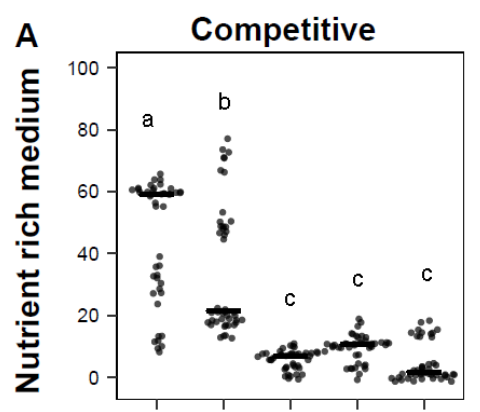

D

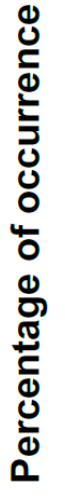
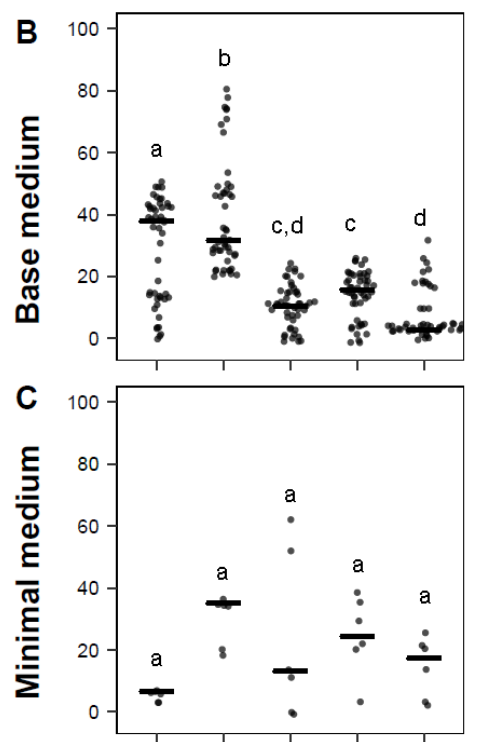

F 1

Metabolite use pattern
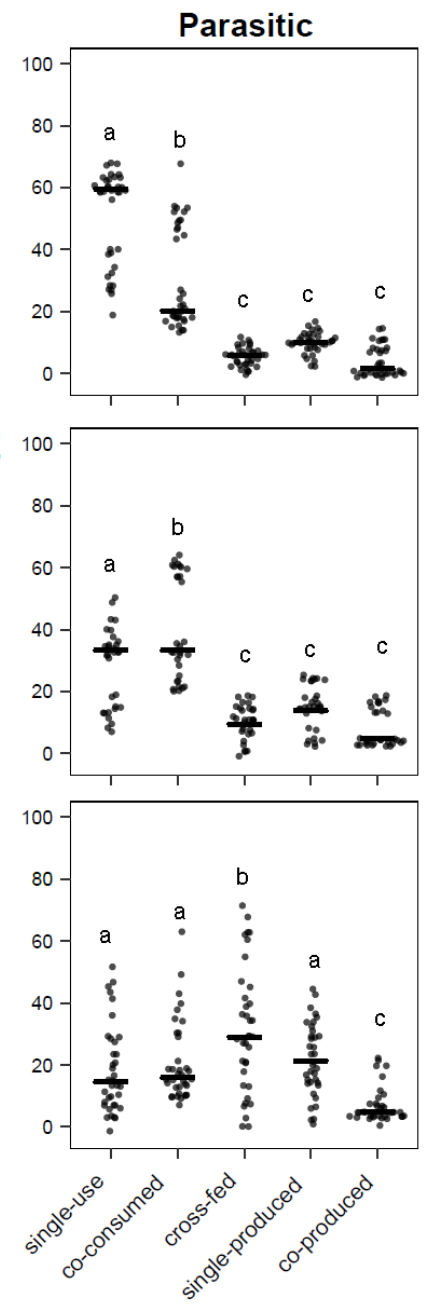

Mutualistic

E 100

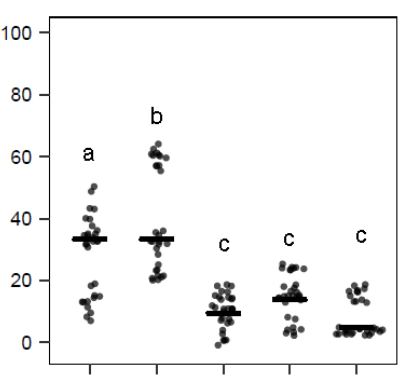

G 10

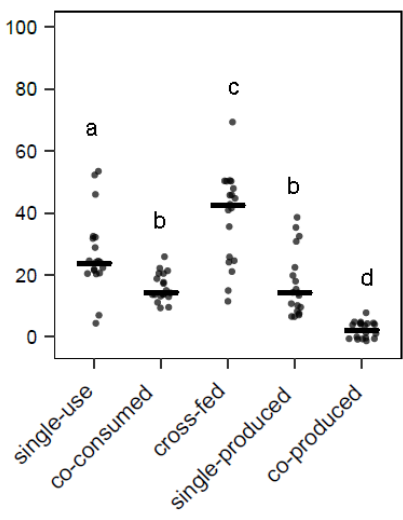


bioRxiv preprint doi: https://doi.org/10.1101/2021.01.20.427455; this version posted January 20, 2021. The copyright holder for this preprint (which was not certified by peer review) is the author/funder. All rights reserved. No reuse allowed without permission.

Fig. 4

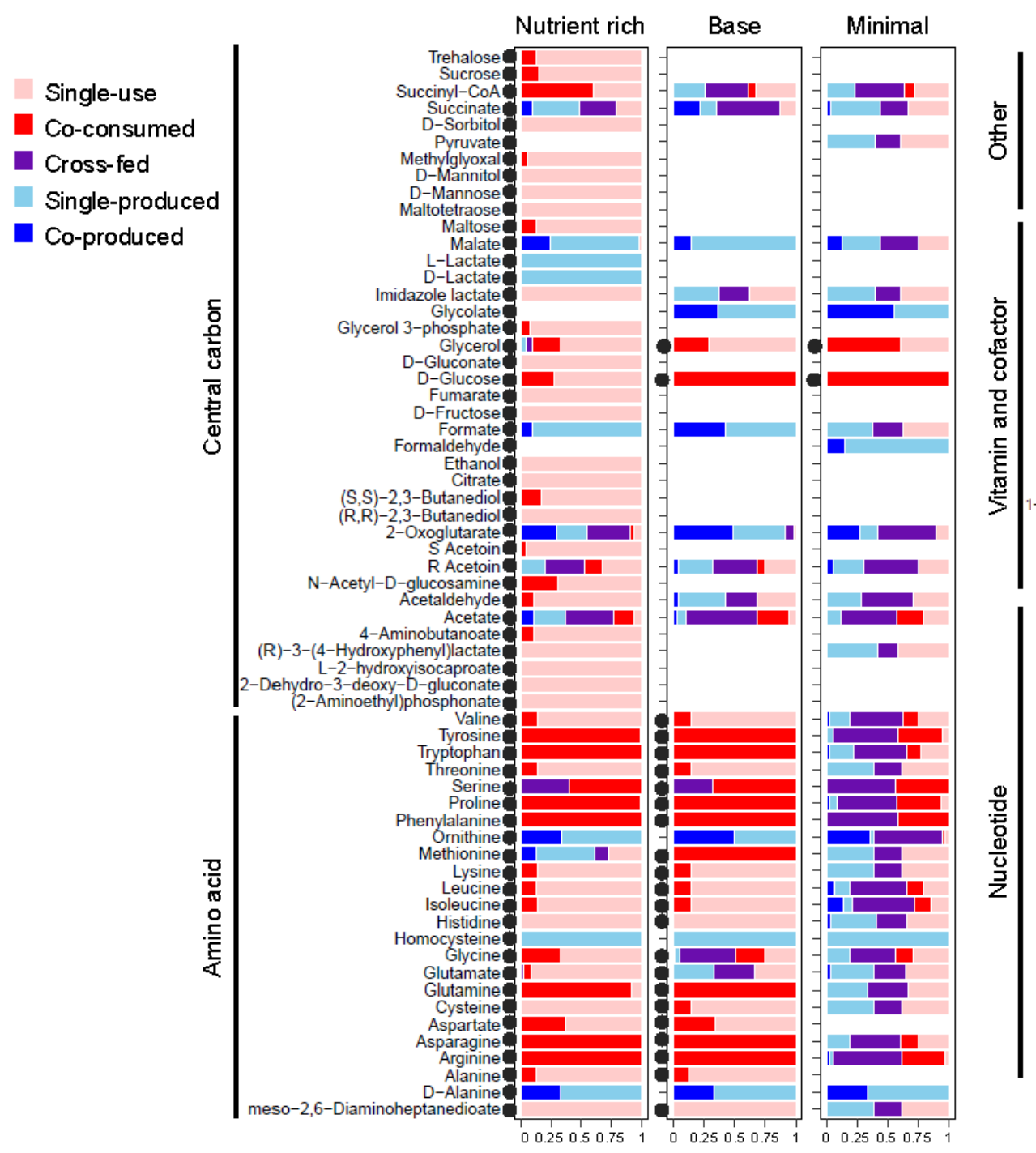

Relative frequency of consumption \& production

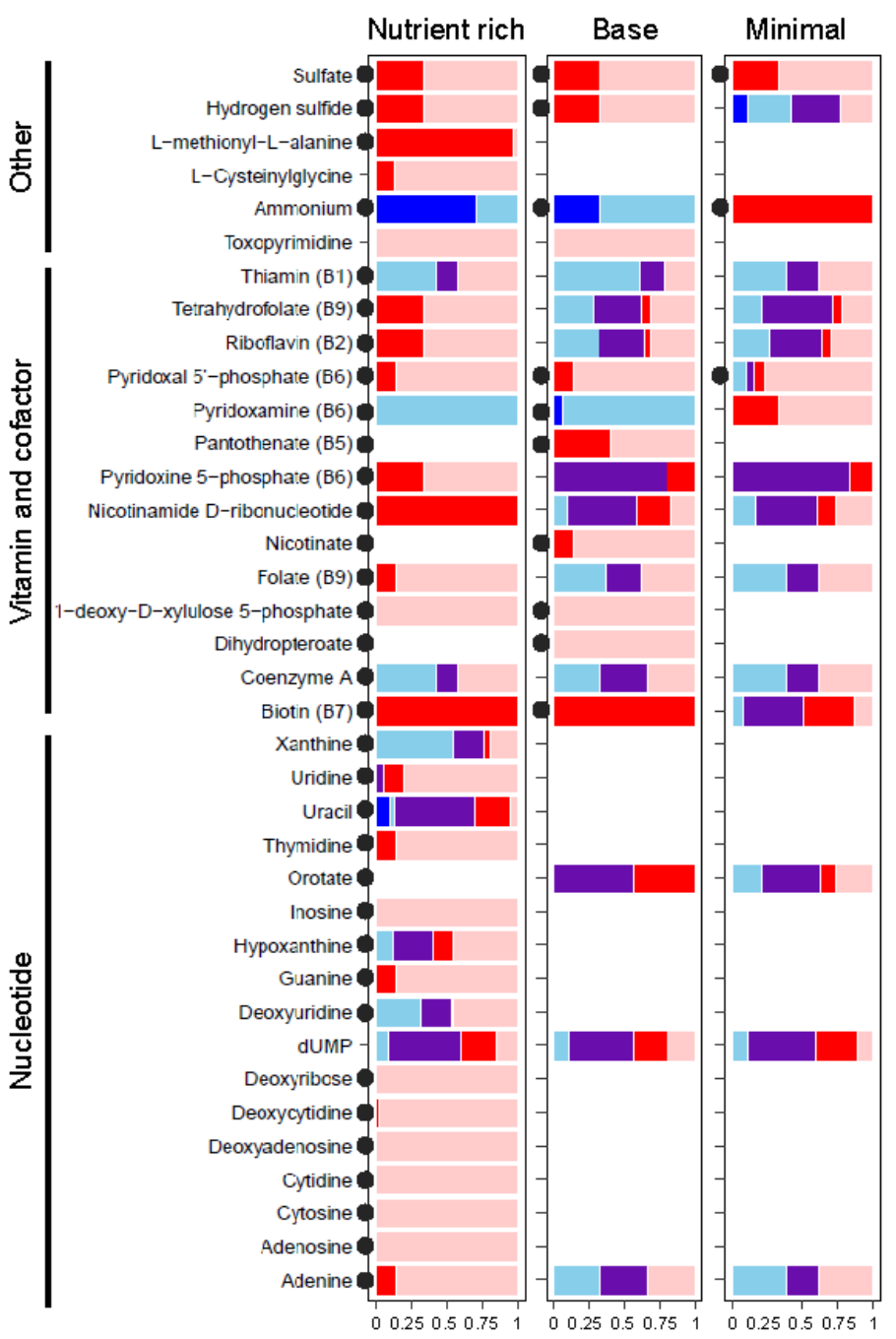

Relative frequency of consumption \& production 
bioRxiv preprint doi: https://doi.org/10.1101/2021.01.20.427455; this version posted January 20, 2021. The copyright holder for this preprint Consume(which was not certified by peer review) is the author/funder. All rights reserved. No reuse allowed without permission.

Nutrient rich medium

AF AP AT LB LP

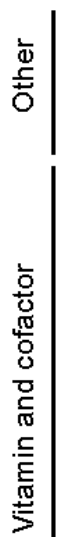

$\frac{\frac{1}{0}}{\frac{0}{0}}$

B

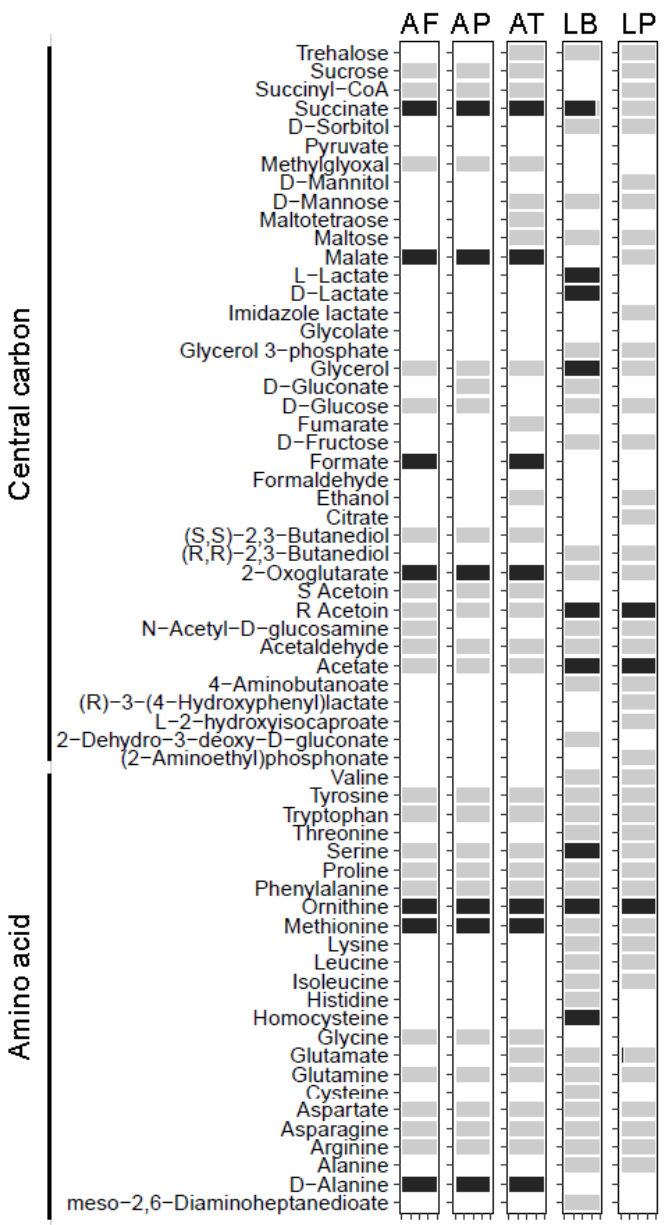

Pyridoxine 5

Nicotinamide D-ribonucleotide

Nicotinate

Folate (B9)

1-deoxy-D-xylulose 5-phosphate

Dihydropteroate

Coenzyme A

Biotin (B7)

Uridine

Uracil

Thymidine

Orotate

Inosine
Hypoxanthine-
Guanine-

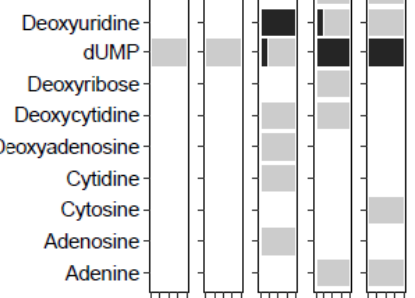

Adenine

trit
Base medium

Minimal medium

AF AP AT LB LP

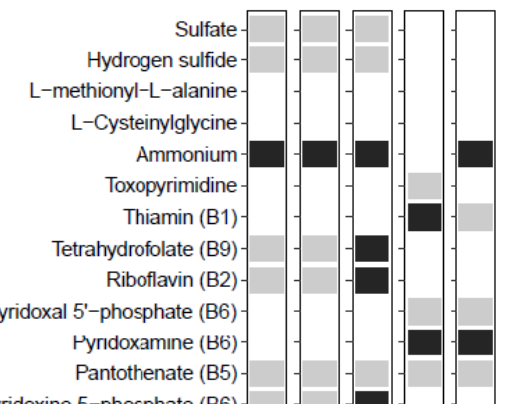

Nicotinamide D-ribonucleotide

Nicotinate

Folate (B9)

1-deoxy-D-xylulose 5-phosphate

Dihydropteroate

Coenzyme A

Biotin (B7)

Xanthine-

Uraci

Thymidin

Inosine

Hypoxanthin

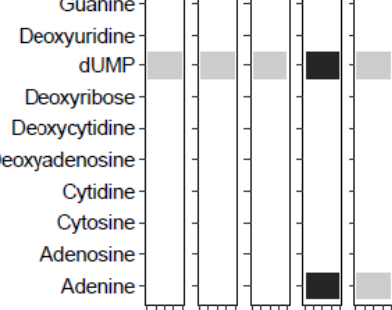

Relative frequency of consumption \& production

Base medium

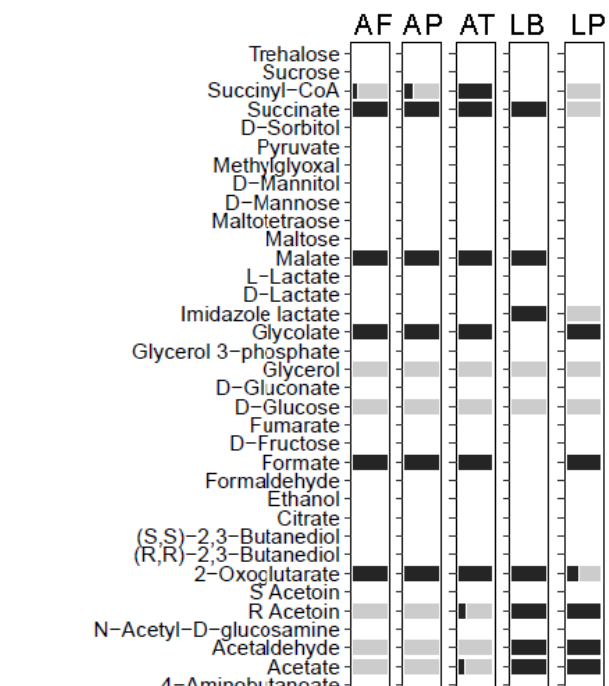

(R)-3-(4-Hydroxyphenyl)lactate

2-Dehydro-3-deoxy-D-gluconate

(2-Aminoethyl)phosphonate

Valine
Tyrosine

Tryptophan

Serine-
Proline-

Phen

Onylalanine-
Ornithine

Methionine

Leysine

Isoleucine

Homocysteine

Glycine
Glutamate

Glutamine

Cysteine
Aspartate

Asparagine-

Arginine-
Alanine-

D-Alanine-

meso-2,6-Diaminoheptanedioate

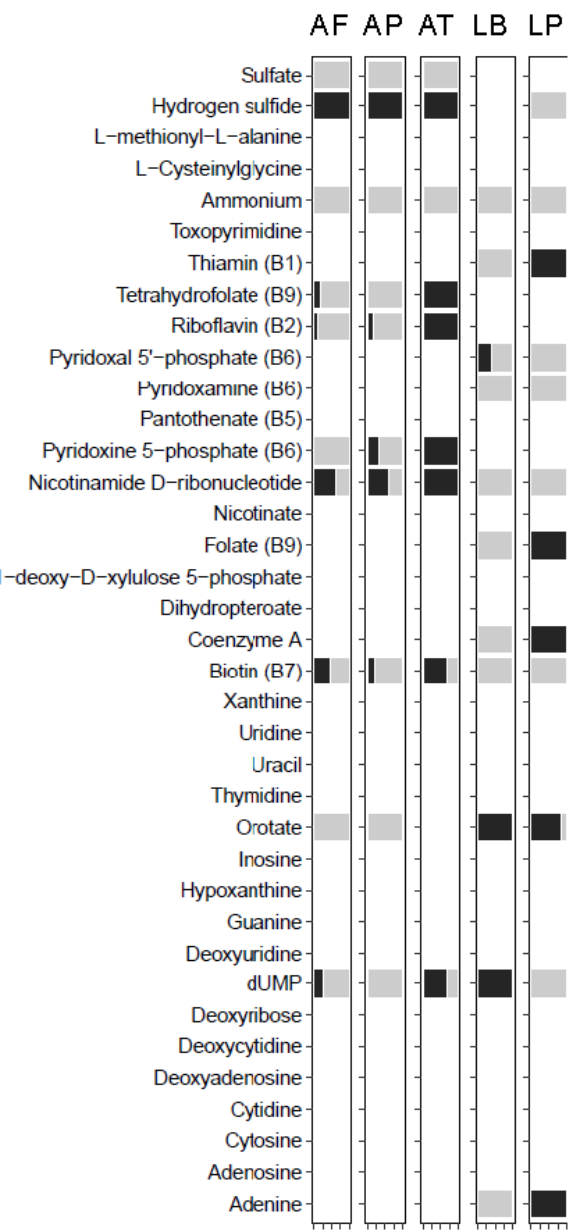

AF AP AT LB LP 
